James $W$. Howse

Lawrence 0 . Ticknor

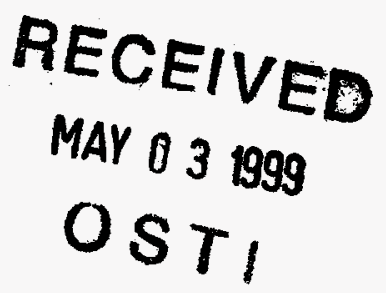

Kenneth R. Muske

Submitted to:

American control Conference

San Diego, CA

June 1999

Los Alamos National Laboratory, an aftirmative actionequal opportunity employer, is operated by the University of California for the U.S. Department of Energy under contract W-7405-ENG-36. By acceptance of this article, the publisher recognizes that the U.S. Government retains a nonexclusive, royalty-free license to publish or reproduce the published form of this contribution, or to allow Government retains a nonexclusive, royalty-free license to publish or reproduce the published form of this contribution, or to allow as work performed under the auspices of the U.S. Department of Energy. The Los Alamos Nationai Laboratory strongly supports academic freedom and a researcher's right to publish; as an institution, however, the Laboratory does not endorse the viewpoint of a publication or guarantee its technical correctness. 


\section{DISCLAIMER}

This report was prepared as an account of work sponsored by an agency of the United States Goverament. Neither the United States Governmeat oor any agency thereof, nor any of their employees, makes any warranty, express or implied, of assumes any legal liability or responsibility for the accuracy, completedess, or usefulness of any information, apparatus, product, or process disclosed, or represents that its use would not infringe privately owned rights. Reference berein to any specific commercial producr, process, or service by trade name, trademark imanufacturer, or otherwise does not necessarily constitute or imply its endorsement, recom. mendation, or favoring by the United States Government or any agency thereof. The views and opinions of authors expressed berein do not necusarily state or reflect those of the United States Governmeat or any agency thercof. 


\section{DISCLAIMER}

Portions of this document may be illegible in electronic image products. Images are produced from the best available original document. 


\title{
Recursive Estimation for the Tracking of Radioactive Sources
}

\author{
James W. Howse \\ Los Alamos National Laboratory, Mail Stop F645 \\ Los Alamos, NM 87545 \\ Lawrence O. Ticknor \\ Los Alamos National Laboratory, Mail Stop F600 \\ Los Alamos, NM 87545 \\ Kenneth R. Muske \\ Dept. of Chemical Engineering, Villanova University \\ Villanova, PA 19085
}

\begin{abstract}
This paper describes a recursive estimation algorithm used for tracking the physical location of radioactive sources in real-time as they are moved around in a facility. The algorithm is related to a nonlinear least squares estimation that minimizes the change in the source location and the deviation between measurements and model predictions simultaneously. The measurements used to estimate position consist of four count rates reported by four different gamma ray detectors. There is an uncertainty in the source location due to the large variance of the detected count rate. This work represents part of a suite of tools which will partially automate security and safety assessments, allow some assessments to be done remotely, and provide additional sensor modalities with which to make assessments.
\end{abstract}

\section{Introduction}

In this paper we describe an algorithm for recursively estimating the real-time positions of radioactive sources in a facility. In this work, we will explicitly consider the problem of tracking a single source within one room. We estimate the position by assuming a known initial source position and then recursively estimating the change in its position from this initial state. Our data is four time series consisting of the count rate at one second intervals from four gamma ray detectors which are located at four different positions within the room. The count rate at a particular sensor is the total number of gammaenergy photons received by the sensor during a one second time interval. The specific sensors that we use consist of a photoluminescent slab of plastic attached to a photomultiplier tube. These sensors do not form an image of the room in the way that a camera would. This means that many of the techniques for locating moving objects in images can not be applied. Our approximations of the detected count rate are based on a nonlinear model of each sensor which relates the source location to the measured count rate at that sensor.

Our algorithm simultaneously minimizes the change in the source location, and the deviation between the measured count rate and the modeled count rate. The procedure can be viewed as a nonlinear least squares estimator which is simultaneously minimizing the expected value of the difference between the detector measurements and the estimated (i.e., model predicted) count rates, and also the expected value of the change in the source location, all in the presence of noise. The predominant source of noise comes from the stochastic nature of gamma emissions from the source itself. Ideally, the emission of gamma photons from a radioactive source over time looks like a series of samples drawn from a Poisson distribution. Since the variance of a Poisson distribution is equal to its mean, the uncertainty in the source location based on the detector readings in a single time interval is fairly high.

An analogy may clarify the difficulties associated with this problem. Imagine a building containing only one room and having a flat roof with four skylights cut into the ceiling, each near one corner of the building. You are standing on the roof and someone is walking around in the room holding a candle. You must determine the position of the candle in the room by observing the relative brightness of the light coming through the four skylights. Keep in mind that the flickering of the candle leads to variations in its brightness that are proportional to the square root of the brightness itself. This analogy makes it clear that many image processing techniques for tracking 
moving objects would be ineffective on this problem.

The facility in question conducts various experiments using radioactive materials, and knowing where the sources are located has implications for both security and safety. Currently all security and safety assessments are made by people who are physically present during experiments. The overall purpose of the project is to provide tools to facilitate and enhance this assessment process. Our algorithm is one part of a suite of tools which will allow some of these assessments to be made remotely. The tools will also provide sensor modalities that are not available to people using only their own senses. These tools will also be used to partially automate the assessment process. These three factors will enhance both security and safety by reducing personnel risk through remote assessment, providing new methods and sensor modalities for risk assessment, and providing a source of independent verification for the current assessment process.

\section{Sensor Model}

In this section we discuss the model that relates the detected count rate from a point source to the position of that source based on the analysis in [Tso83]. For the $i$ th detector, the relationship between the detected count rate $\mathcal{D}_{i}$ and the source position $\left(u_{i}, v_{i}, w_{i}\right)$ relative to that detector is given by

$$
\begin{aligned}
& \mathcal{D}_{i}=\frac{\Omega_{i}\left(u_{i}, v_{i}, w_{i}\right) \mathcal{S} \epsilon_{i} \mathcal{F}_{i}+\mathcal{B}_{i}}{1+\tau_{i} \Omega_{i}\left(u_{i}, v_{i}, w_{i}\right) \mathcal{S} \epsilon_{i} \mathcal{F}_{i}} \\
& \Rightarrow \mathcal{D}_{i}=\mathcal{M}_{i}\left(u_{i}, v_{i}, w_{i}\right)
\end{aligned}
$$

for $i=1,2, \ldots, m$, where in our case $m=4$. In this equation $\mathcal{S}$ is the actual source strength, $\epsilon_{i}$ is the detector efficiency, $\tau_{i}$ is the dead time, $\mathcal{F}_{i}$ is the product of all the correction factors (e.g., absorption and backscattering), $\mathcal{D}_{i}$ is the total number of counts per unit time actually detected, $\mathcal{B}_{i}$ is the number of counts per unit time which constitute the background, and the view factor $\Omega_{i}(\cdot)$ is the ratio of the number of particles which actually enter the detector to the total number of particles emitted by the source. The quantity $\Omega_{i}(\cdot)$ can also be thought of as the solid angle subtended by the detector for a particular source location, which ranges between 0 and $4 \pi$. For these detectors, the efficiency is rated at $\sim 10 \%$ for a $\mathrm{Cs}^{137}$ source. The dead time of a detector is defined to be the minimum length of time which must separate incident photons in order for them to be recorded as two separate pulses. The photoluminescent material used in these detectors has a dead time of 3.3 nanoseconds. Note that there is one equation of this form for each of the $m$ detectors. We will use the right hand side of Equation (1) as an approximation for the count rate given a particular position $\left(u_{i}, v_{i}, w_{i}\right)$, and we denote this function by $\mathcal{M}_{i}(\cdot)$.
For a point source and a rectangular detector of finite size, the most general relative position is shown in Figure 1 . The solid angle $\Omega_{i}(\cdot)$ subtended by a

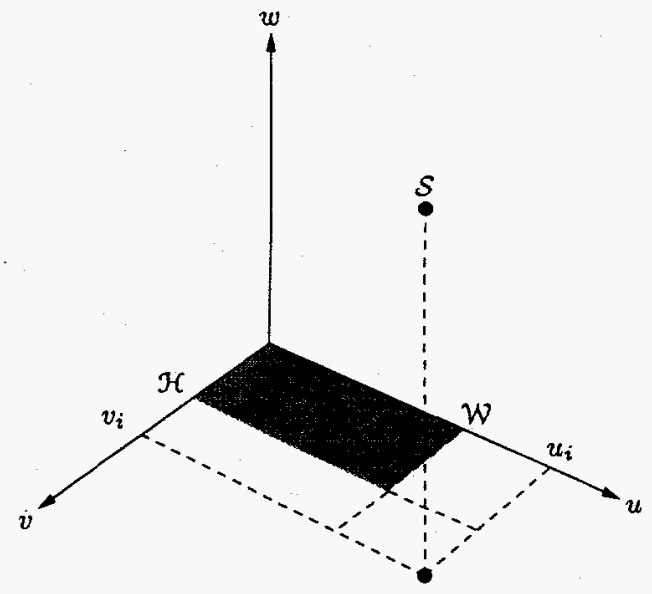

Figure 1: The relative position between a point source at position $\mathcal{S}$ and a rectangular detector of width $\mathcal{W}$ and height $\mathcal{H}$. The projections of the source location onto the $u$ and $v$ axes are $u_{i}$ and $v_{i}$ respectively.

detector of width $\mathcal{W}$ and height $\mathcal{H}$ for a point source located at $\left(u_{i}, v_{i}, w_{i}\right)$, relative to the $i$ th detector, is given by

$$
\begin{array}{r}
\Omega_{i}\left(u_{i}, v_{i}, w_{i}\right)=\tan ^{-1}\left(\frac{u_{i} v_{i}}{w_{i} \sqrt{u_{i}^{2}+v_{i}^{2}+w_{i}^{2}}}\right) \\
-\tan ^{-1}\left(\frac{\left(u_{i}-\mathcal{W}\right) v_{i}}{w_{i} \sqrt{\left(u_{i}-\mathcal{W}\right)^{2}+v_{i}^{2}+w_{i}^{2}}}\right) \\
-\tan ^{-1}\left(\frac{u_{i}\left(v_{i}-\mathcal{H}\right)}{w_{i} \sqrt{u_{i}^{2}+\left(v_{i}-\mathcal{H}\right)^{2}+w_{i}^{2}}}\right) \\
+\tan ^{-1}\left(\frac{\left(u_{i}-\mathcal{W}\right)\left(v_{i}-\mathcal{H}\right)}{w_{i} \sqrt{\left(u_{i}-\mathcal{W}\right)^{2}+\left(v_{i}-\mathcal{H}\right)^{2}+w_{i}^{2}}}\right)
\end{array}
$$

as shown in [GY71]. The detectors that were used have a height $\mathcal{H}$ of 0.83 feet and a width $\mathcal{W}$ of 3.00 feet.

It is clear from Figure 1 that the coordinate system used to derive Equation (2) is detector centered, not room centered. Since the desired answer is the source location in room centered coordinates, a transformation must be made between these two coordinate systems. The required transformation converts room centered coordinates $(x, y, z)$ into detector centered coordinates $\left(u_{i}, v_{i}, w_{i}\right)$, which allows Equation (2) to be properly evaluated. One way to construct this transformation is by moving each of the detectors from their actual location to the selected origin of the room. This is accomplished by first rotating the sensor about the $v$-axis in Figure 1, and then translating the rotated sensor to the origin of the room. 
This transformation can be written in matrix form as

$$
\left(\begin{array}{c}
u_{i} \\
v_{i} \\
w_{i} \\
1
\end{array}\right)=\left(\begin{array}{cccc}
\cos \mathcal{A}_{i} & 0 & \sin \mathcal{A}_{i} & \mathcal{T}_{x, i} \cos \mathcal{A}_{i}+ \\
0 & 1 & 0 & \mathcal{T}_{z, i} \sin \mathcal{A}_{i} \\
\mathcal{T}_{y, i} \\
-\sin \mathcal{A}_{i} & 0 & \cos \mathcal{A}_{i} & \mathcal{T}_{z, i} \cos \mathcal{A}_{i}- \\
0 & 0 & 0 & \mathcal{T}_{x, i} \sin \mathcal{A}_{i} \\
0 & & 1
\end{array}\right)\left(\begin{array}{l}
x \\
y \\
z \\
1
\end{array}\right),
$$

where $\mathcal{T}_{x, i}, \mathcal{T}_{y, i}$, and $\mathcal{T}_{z, i}$ are the translations along the room centered $x, y$, and $z$ directions, respectively, for the $i$ th detector. The quantity $\mathcal{A}_{i}$ is the rotation angle around the $v$-axis for the $i$ th detector. Note that the source location in room centered coordinates is $(x, y, z)$, and that the source strength $\mathcal{S}$ is not changed by the transformation in Equation (3).

\section{Estimation Algorithm}

In this section we discuss the estimation algorithm used to track the position of the radioactive source over time. This problem was posed as a state estimation problem whose formulation is explained, for instance, in [SM71] and [Jaz70]. In this context the measurements or outputs are the detected count rate $\mathcal{D}_{i}(k)$ at each detector $i$ at each time step $k$. The states are chosen according to the problem that must be solved. In this case, the state must at least contain the source location $(x(k), y(k), z(k))$ at every time step $k$. In addition to the source location, another unknown in Equation (1) is the background count rate $\mathcal{B}_{i}$ at each detector. We will assume that the background is the same at each detector, making $\mathcal{B}(k)$ independent of $i$, and include this single quantity in the state. We assume that the source strength $\mathcal{S}$ is known. We also assume that at time $k=0$ we have a good estimate of the source position and background level $(x(0), y(0), z(0), \mathcal{B}(0))$. Lastly we assume that the correction factor product $\mathcal{F}_{i}$ is constant over time. Note that while these assumptions are not always reasonable, in our problem they are often valid.

At succeeding time steps we update our estimate of the source location and background level using the relations

$$
\begin{aligned}
& x(k)=x(k-1)+\delta x^{*}(k), \\
& y(k)=y(k-1)+\delta y^{*}(k), \\
& z(k)=z(k-1)+\delta z^{*}(k), \\
& \mathcal{B}(k)=\mathcal{B}(k-1)+\delta \mathcal{B}^{*}(k),
\end{aligned}
$$

for all time steps $k=1,2,3, \ldots$, where $\delta x^{*}(k)$, $\delta y^{*}(k), \delta z^{*}(k)$, and $\delta \mathcal{B}^{*}(k)$ are state changes which are optimal in some sense. Denote any change in the state estimates at time $k$ by the vector $d(k)=$ $[\delta x(k) \delta y(k) \delta z(k) \delta \mathcal{B}(k)]$, denote the state estimates at a particular time step $k$ by the vector $e(k)=$ $[x(k) y(k) z(k) \mathcal{B}(k)]$ and call the number of elements in each of these vectors $p=4$. Using these definitions, Equation (4) can be written in vector form as $\boldsymbol{e}(k)=\boldsymbol{e}(k-1)+\boldsymbol{d}^{*}(k)$. At each time step $k$ the optimal estimated state change $\boldsymbol{d}^{*}(k)$ is computed by solving the optimization problem

$$
\begin{array}{r}
\boldsymbol{d}^{*}(k)=\arg \min _{\boldsymbol{d}(k)}\left(\sum _ { i = 1 } ^ { \mathrm { m } } \mathcal { W } _ { i } ^ { m } \left(\mathcal{D}_{i}-\mathcal{M}_{i}(\boldsymbol{e}(k-1)\right.\right. \\
\left.+\boldsymbol{d}(k)))^{2}+\sum_{j=1}^{\mathrm{p}} \mathcal{W}_{j}^{d} d_{j}^{2}(k)\right)
\end{array}
$$

subject to

$$
\begin{gathered}
-\mathcal{B}_{j} \leq d_{j}(k) \leq \mathcal{B}_{j}, \\
\mathcal{L}_{j} \leq e_{j}(k-1)+d_{j}(k) \leq \mathcal{U}_{j}, \quad j=1, \ldots, p .
\end{gathered}
$$

In this equation, $\mathcal{M}_{i}(e(k-1)+d(k))$ represents our approximation of the detected count rate $\hat{\mathcal{D}}_{i}$, given by the right hand side of Equation (1) evaluated at $e(k-1)+d(k)$ for the $i$ th detector. Note that evaluating the right hand side of Equation (1) requires solving Equations (2) and (3) first. The quantity $\mathcal{W}_{i}^{m}$ is a weight which determines how closely the algorithm tries to match the model. Similarly, $\mathcal{W}_{j}^{d}$ sets how much the algorithm tries to change the previous state estimate. The term $\sum_{i=1}^{m} \mathcal{W}_{i}^{m}\left(\mathcal{D}_{i}-\mathcal{M}_{i}(e(k-\right.$ 1) $+\boldsymbol{d}(k)))^{2}$ is a weighted $\mathcal{L}_{2}$ norm of the difference $\|\mathcal{D}-\hat{\mathcal{D}}\|_{2}$ between the actual detected count rate $\mathcal{D}$ and the predicted detected count rate $\hat{\mathcal{D}}$. Likewise the term $\sum_{j=1}^{p} \mathcal{W}_{i}^{d} d_{j}^{2}(k)$ is the weighted $\mathcal{L}_{2}$ norm of the difference $\|\boldsymbol{e}(k)-e(k-1)\|_{2}$ between the estimated position and background at the previous time and that at the current time. Conceptually this is a regularization term which penalizes large changes in the state estimate more than small changes. A block diagram of this estimation algorithm is shown in Figure 2. In this figure, $\mathcal{M}(\cdot)$ is the vector form for

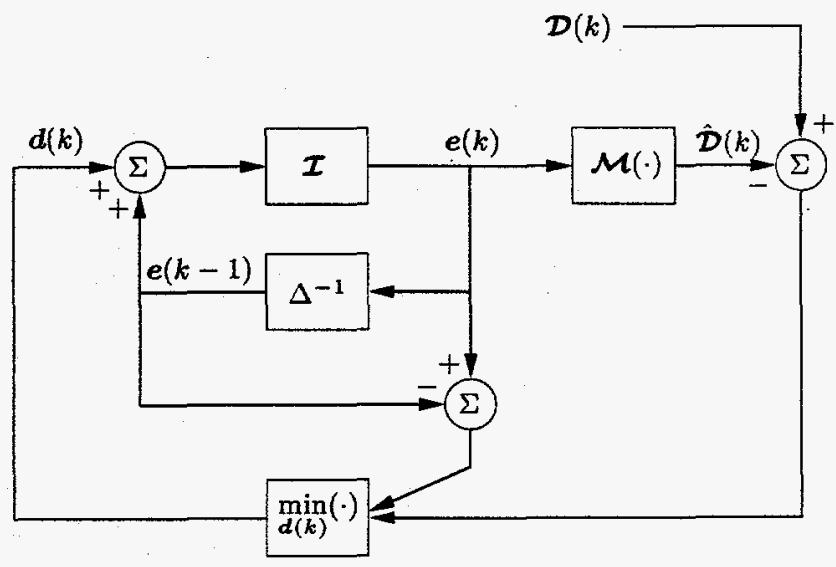

Figure 2: A block diagram of the state estimation procedure.

the right hand side of Equation (1), $\mathcal{I}$ is the identity mapping, $\Delta^{-1}$ is a unit time delay, and $\min _{d(k)}(\cdot)$ 
is the optimization problem in Equation (5). So Equation (5) will try to minimize the change in position and background while simultaneously making a change that makes the resulting position and background approximately agree with the model. When selecting the weights, note that making $\mathcal{W}_{i}^{m}$ large relative to $\mathcal{W}_{i}^{d}$ reflects a high confidence in the sensor model, while setting $\mathcal{W}_{i}^{d}$ high with respect to $\mathcal{W}_{i}^{m}$ indicates confidence in the state estimate.

Note that there are upper and lower bounds on the state change $\boldsymbol{d}(k)$ and on the state estimate $\boldsymbol{e}(k)$. The constraints on the estimate $e(k)$ are imposed so that the estimated position can not be outside the room, and the estimated background can not exceed historical bounds for the background levels in the room. The constraints on the change in estimate $d(k)$ are imposed in order to keep noise from causing the position to jump around excessively from time step to time step. Conceptually these constraints are reasonable because the sources are moved by people, and there is a limit to how far a person can move in a given time interval.

The optimization problem posed in Equation (5) is a nonlinear programming problem with linear inequality constraints, which can be solved by a number of methods, many of which are discussed in [Ber95]. The optimization algorithm that we chose was CFSQP, which is documented in [LZT94]. This algorithm uses a sequential quadratic programming (SQP) approach, modified so that each iteration is feasible with respect to the constraints. We chose this algorithm because all the intermediate iterates of the algorithm are feasible, so if we have to stop the optimization before achieving convergence, the resulting suboptimal solution will still satisfy the constraints.

\section{Tracking Results}

In this section we present some tracking results based on experiments with a real source in the facility. The experimental procedure consisted of placing a $\mathrm{Cs}^{137}$ source on a cart and moving it along a predetermined path in the room. Along this path there were 17 points at which we paused with the source for approximately 3 minutes each. The estimated position of the source as seen from the ceiling looking down is shown in Figure 3 . Figure 3(a) shows the estimated source positions for model weights $\mathcal{W}_{i}^{m}=10^{-4}$ for all $i$, and Figure 3(b) shows the locations with $\mathcal{W}_{i}^{m}=10^{-6}$. In both cases the all the state weights were $\mathcal{W}_{j}^{d}=1$. The 17 numbers in these plots show the actual positions of the points at which we paused with the source. Beginning at point 1 , we followed the numbered points in ascending order, ending at point 17 . The points - in the figure represent the pairs $(x(k), z(k))$ of the state estimate for every time step $k$. These estimates are computed at 1 second intervals, which is also the sampling rate for the detectors. Our algorithm runs

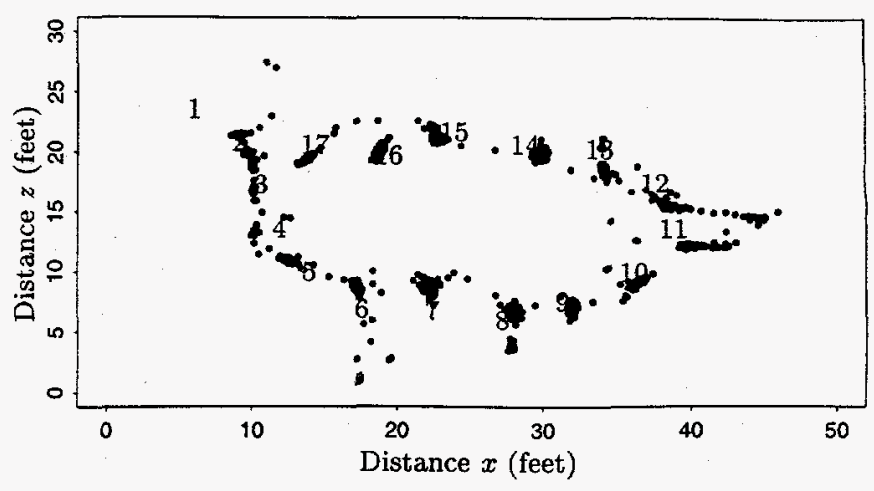

(a)

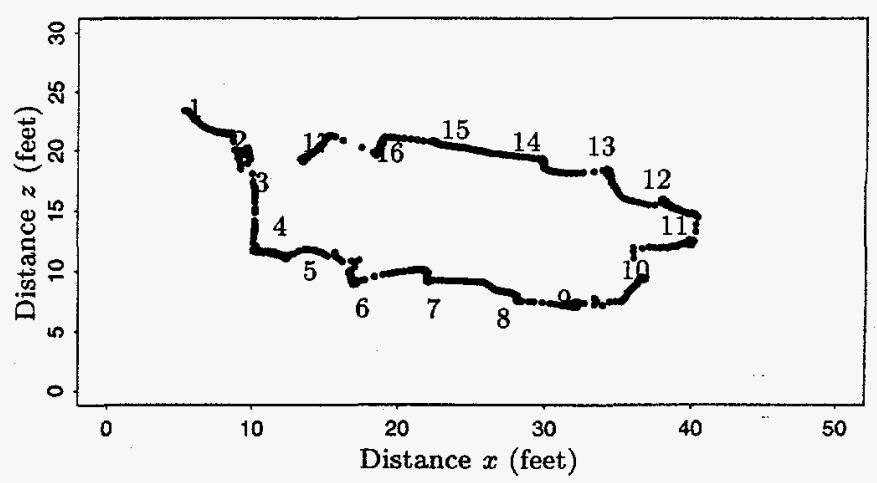

(b)

Figure 3: The estimated position of the source in the $x-z$ plane with model weights (a) $\mathcal{W}_{i}^{m}=10^{-4}$ (b) $\mathcal{W}_{i}^{m}=$ $10^{-6}$. These plots show a view of the room from the ceiling looking down. The detectors are located in each of the four corners of these pictures.

fast enough for us to compute each state estimate in less than 1 second, hence we are able to track the source in real-time relative to the detector sampling rate. Although the detectors do not appear in these pictures, they are located in each corner at a $45^{\circ}$ angle with respect to the walls. Note that in Figure 3(a) most of the position estimates cluster around one of the 17 numbered points, while in Figure 3(a) there is a smooth track of points running through the 17 numbered points.

Figure 3 shows that most of the estimates of the source location are close to the path described by the 17 points where we paused with the source. However, it does not show. whether the estimates follow this path in the correct temporal order. Figure 4 shows the estimated position in both the $x$ and $z$ directions versus time for the $x-z$ plot shown in Figure 3(a). In these two plots the the solid lines represent the estimated source position, and the dotted lines represent the actual source position. Figure 4(a) plots the position in the $x$ direction versus time, and Figure 4(b) 


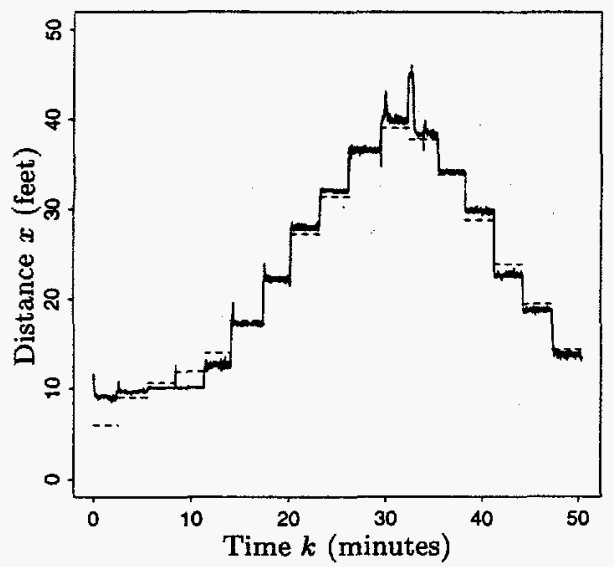

(a)

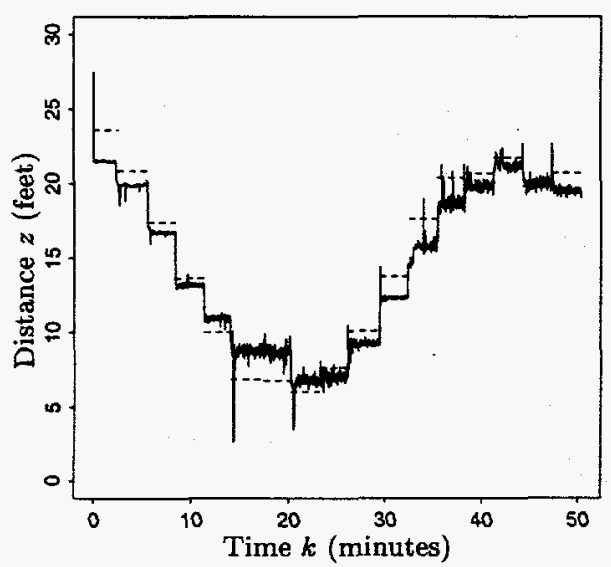

(b)

Figure 4: The estimated position of the source in the (a) $x$-direction (b) $z$-direction versus time. The dotted lines show the actual source position over time.

shows the $z$ position with respect to time. It is clear from this figure that the numbered points are visited in the correct order.

We estimated the error of this tracking algorithm in the following way. For each plateau in Figure 4(a) compute the absolute value of the difference between the median estimated position and the actual position. Then take the maximum value of this difference over all the plateaus. We performed the same calculation for the data in Figure 4(b). This gives a maximum median error in both the $x$ and $z$ directions. Note that this error measure is probably dependent on the source strength. For the data shown in Figure 4 this error was 3.11 feet in the $x$ direction and 2.14 feet in the $z$ direction. So the maximum median exror leads to an uncertainty in the source location which is a rectangle around the actual location that is 6.22 feet in the $x$ direction and 4.28 feet in the $z$ direction. Dividing the area of this rectangle by the area of the room itself leads to a percent location error of $2.10 \%$. For the $x-z$ points shown in Figure 3(b), this error statistic is $1.70 \%$. In achieving this higher accuracy, the trade-off is that the estimated location lags the actual location in time. So there is a tradeoff between accuracy in space and accuracy in time for this estimation algorithm.

\section{Conclusion}

We have described a real-time algorithm for tracking the position of radioactive sources in a facility in the presence of measurement noise. We formulated this problem as a state estimation problem and solved it recursively using a constrained nonlinear optimization method. The optimization simultaneously minimizes the change in source position and disagreement between measurements and a sensor model. The sensor model is a fairly complex function relating position to detected count rate. The overall purpose of this work is to enhance both security and safety by automating part of the assessment process, allowing remote assessment, and introducing new sensor modalities into the assessment process. We presented tracking results based on an experiment done with one source in a single room. Our results indicate that a source can be tracked quite well with this algorithm in spite of rather high measurement noise levels. In short, we have demonstrated the capability to track a single source in real-time with high accuracy in spite of a complex sensor model and high measurement noise.

\section{References}

[Ber95] D.P. Bertsekas. Nonlinear Programming. Optimization and Neural Computation Series. Athena Scientific, Belmont, MA, 1995.

[GY71] H. Gotoh and H. Yagi. Solid angle subtended by a rectangular slit. Nuclear Instruments and Methods, 96(2):485-486, 1971.

[Jaz70] A.H. Jazwinski. Stochastic Processes and Filter Theory, volume 64 of Mathematics in Science and Engineering. Academic Press, Inc., New York, NY, 1970.

[LZT94] C.T. Lawrence, J.L. Zhou, and A.L. Tits. User's guide for CFSQP. Technical Report TR-9416r1, Institute for Systems Research, University of Maryland, College Park, MD, 1994.

[SM71] A.P. Sage and J.L. Melsa. Estimation Theory with Applications to Communications and Control. Systems Science Series. McGraw-Hill, Inc., New York, NY, 1971.

[Tso83] N. Tsoulfanidis. Measurement and Detection of Radiation. Nuclear Engineering Series. McGrawHill, Inc., New York, NY, 1983. 